\title{
Nonsurgical maxillary expansion in a 60-year-old patient with gingival recession and crowding
}

Harim Kim ${ }^{\mathrm{a}}$ (1)

Sun-Hyung Park ${ }^{\mathrm{a}}$

Jae Hyun Park ${ }^{\mathrm{b}}$

Kee-Joon Lee ${ }^{\mathrm{a}}$ (1)

aDepartment of Orthodontics, Institute of Craniofacial Deformity, Yonsei University College of Dentistry, Seoul, Korea

${ }^{\mathrm{b}}$ Postgraduate Orthodontic Program, Arizona School of Dentistry \& Oral Health, A. T. Still University, Mesa, AZ, USA
Maxillary transverse deficiency often manifests as a posterior crossbite or edgeto-edge bite and anterior crowding. However, arbitrary arch expansion in mature patients has been considered to be challenging due to the possible periodontal adverse effects such as alveolar bone dehiscence and gingival recession. To overcome these limitations, nonsurgical maxillary expansion of the basal bone has been demonstrated in young adults. However, the age range for successful orthopedic expansion has remained a topic of debate, possibly due to the underlying individual variations in suture maturity. This case report illustrates nonsurgical, miniscrew-assisted rapid palatal expansion (MARPE) in a 60-yearold patient with maxillary transverse deficiency accompanied by anterior and posterior crossbites, crowding, and gingival recession. The use of MARPE allowed relief of crowding and correction of the crossbite without causing significant periodontal adverse effects.

[Korean J Orthod 2021;51(3):217-227]

Key words: Nonsurgical palatal expansion, Miniscrew-assisted rapid palatal expansion, Adult treatment, Gingival recession
Received August 25, 2020; Revised October 20, 2020; Accepted November 25, 2020.

Corresponding author: Kee-Joon Lee.

Professor, Department of Orthodontics, Institute of Craniofacial Deformity, Yonsei University College of Dentistry, 50-1 Yonsei-ro, Seodaemun-gu, Seoul 03722, Korea.

Tel +82-2-2228-3105 e-mail orthojn@yuhs.ac

How to cite this article: Kim H, Park SH, Park JH, Lee KJ. Nonsurgical maxillary expansion in a 60-year-old patient with gingival recession and crowding. Korean $\mathrm{J}$ Orthod 2021;51:217-227.

(C) 2021 The Korean Association of Orthodontists.

This is an Open Access article distributed under the terms of the Creative Commons Attribution Non-Commercial License (http://creativecommons.org/licenses/by-nc/4.0) which permits unrestricted non-commercial use, distribution, and reproduction in any medium, provided the original work is properly cited. 


\section{INTRODUCTION}

Crowding can be managed in a number of different ways, including arch expansion, flaring of the anterior teeth, and distalization. In the absence of significant anteroposterior discrepancies such as in Class 11 or Class 111 molar relationships, a transverse deficiency may be suspected to be a major cause of crowding. Transverse expansion can be achieved by dentoalveolar displacement using a transpalatal arch (TPA) or expansion archwire. Since TPAs and expansion archwires largely result in buccal tipping of the posterior teeth, ${ }^{1}$ they are not readily used unless the molars are lingually tipped. Furthermore, dentoalveolar expansion can cause undesirable periodontal side effects. The reduced periodontal support can be functionally and esthetically detrimental.

Displacement of the premolars and/or molars to the buccal side is mostly contraindicated, especially in aged patients, due to the risk of gingival recession and bony dehiscence. Even in the absence of active periodontal lesions, alveolar bone loss is a natural consequence of aging. ${ }^{2,3}$ Therefore, studies reporting the use of active expansion in aged patients are scarce in orthodontic literature.

In patients with maxillary transverse deficiency, skeletal expansion offers a good therapeutic option without the need for periodontal compromise, while maintaining the buccolingual axis of the posterior segment. Angell ${ }^{4}$ first introduced the idea of rapid palatal expansion (RPE) in 1860 , which was again demonstrated by Haas ${ }^{5}$ in the 1960s mainly in around the pubertal stage. Moreover, successful orthopedic expansion using miniscrew-as- sisted RPE (MARPE) has been shown in young adults. ${ }^{6,7}$ Nonetheless, skeletal maturity and the increasing interdigitation of the facial sutures are known to be the main limiting factors for RPE. ${ }^{8,9}$ Accordingly, surgically assisted RPE (SARPE) has been suggested as an alternative to overcome the mechanical resistance to suture opening in adult patients. ${ }^{10,11}$ Meanwhile, the age range for successful orthopedic expansion has remained a topic of debate, possibly due to the underlying individual variations in suture maturity.

Unlike calvarial sutures, which are known to fuse at around the 20s, the facial sutures have been shown to remain patent after cessation of active growth. ${ }^{12}$ This indicates the possibility of successful orthopedic expansion even in skeletally mature and old patients. The following case describes the treatment of a 60-year old patient who showed crowding accompanied by the risk of gingival recession and alveolar bone loss via nonsurgical orthopedic expansion.

\section{DIAGNOSIS AND ETIOLOGY}

A 60-year old female patient presented with the chief complaint of unaesthetic smile involving a crossbite and crowding of the anterior teeth. She had a history of previous orthodontic treatment due to crowding approximately 50 years ago. The intraoral examination indicated an anterior crossbite of the left lateral incisors and a posterior crossbite of the left second premolars, as well as an edge-to-edge bite of the left first premolars. She had a symmetrical Class 1 molar relationship. The dental midline deviated $2 \mathrm{~mm}$ to the left in the

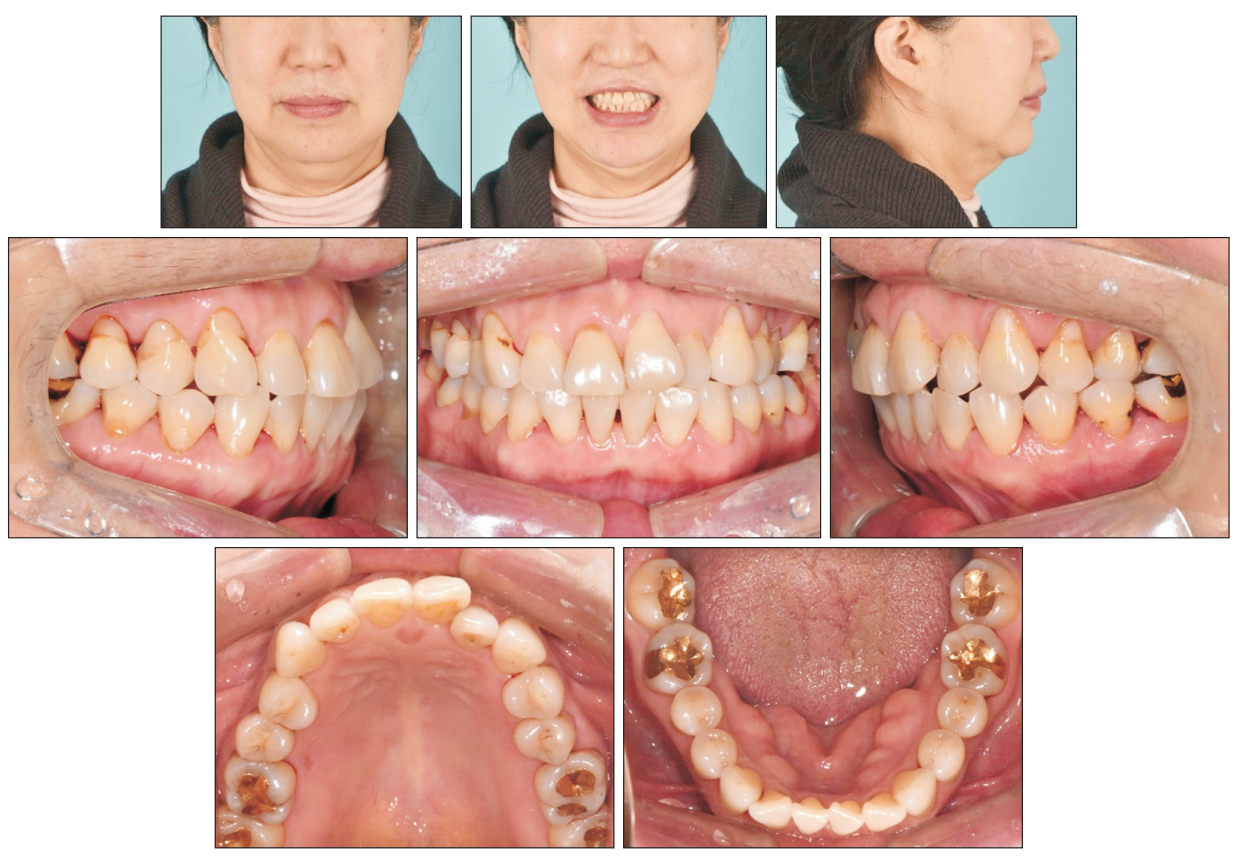

Figure 1. Pretreatment facial and intraoral photographs. 
maxilla and $1 \mathrm{~mm}$ to the left in the mandible relative to the facial midline (Figure 1). The intermolar width, which was measured as the distance between the central fossa of the right and left first molars, was $47.0 \mathrm{~mm}$ in the maxilla and $43.0 \mathrm{~mm}$ in the mandible, whereas the normal intermolar width difference ranged from 5 to 8 $\mathrm{mm} .{ }^{13,14}$ The interpremolar width was $34.0 \mathrm{~mm}$ in the maxilla and $32.6 \mathrm{~mm}$ in the mandible. The arch widths were also measured at the estimated centers of resistance (middle point of furcation). ${ }^{14}$ The difference was $-4.0 \mathrm{~mm}$, with the widths being $44.1 \mathrm{~mm}$ in the maxilla and $48.1 \mathrm{~mm}$ in the mandible. This was in contrast to the reported mean value in normal occlusion, which is $-0.39 \pm 1.87 \mathrm{~mm} .{ }^{14}$ Accordingly, the basal arch width of the maxilla was considered as relatively narrow. Further assessment of the diagnostic data revealed a mild hyperdivergence (skeletal Class 11) with maxillary and mandibular arch length discrepancies amounting to 3 and 2 $\mathrm{mm}$, respectively (Figure 2, Table 1 ). The patient showed some generalized horizontal alveolar bone loss and noninflammatory gingival recession, especially on the facial surfaces of the upper left central incisor, canine, and premolars. Furthermore, a predisposition to cervical abfraction could be inferred from the presence of multiple cervical fillings (Figure 1).

Informed consent of the patient was obtained for release of the patient's records.

\section{TREATMENT OBJECTIVES}

The treatment objectives were (1) to correct the anterior and posterior crossbites, (2) to relieve the maxillary and mandibular crowding without any change in the profile, (3) to maintain the bilateral Class 1 molar relationship, (4) to maintain periodontal health by avoiding further buccal bone loss and gingival recession, and (5) to improve the smile esthetics. Considering the advanced age of the patient and the presence of periodontal issues, the treatment was kept as minimally invasive and short as possible.

\section{TREATMENT ALTERNATIVES}

Based on the diagnosis, several treatment options were suggested. Flaring of the anterior teeth provides space for crowding relief; however it results in increasing convexity of the facial profile. Since the patient did not want any change in the profile, this treatment option

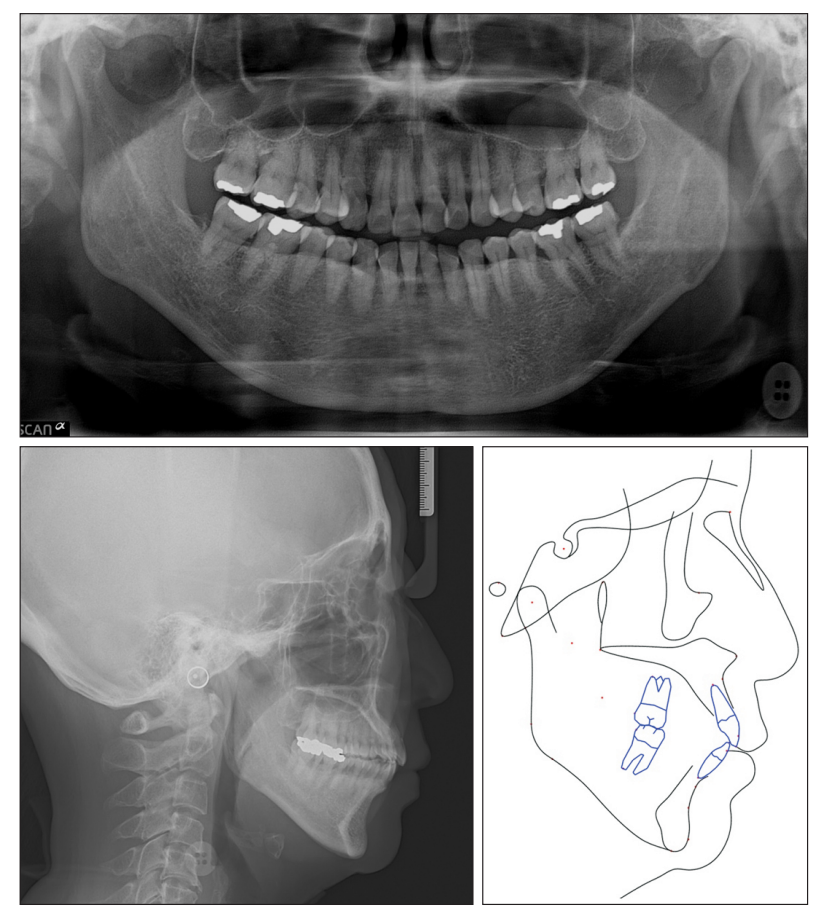

Figure 2. Pretreatment radiographs and cephalometric tracing.

Table 1. Cephalometric analysis

\begin{tabular}{lccc}
\hline \multicolumn{1}{c}{ Measurement } & Mean & Pretreatment & Posttreatment \\
\hline SNA $\left(^{\circ}\right)$ & 81.6 & 75.2 & 75.4 \\
SNB $\left(^{\circ}\right)$ & 79.1 & 70.6 & 70.6 \\
ANB $\left(^{\circ}\right)$ & 2.4 & 4.6 & 4.8 \\
Wits $(\mathrm{mm})$ & -2.8 & 1.3 & 1.7 \\
SN-GoMe $\left(^{\circ}\right)$ & 34.0 & 50.2 & 50.0 \\
U1 to SN $\left(^{\circ}\right)$ & 106.0 & 97.7 & 96.3 \\
L1 to GoGn $\left(^{\circ}\right)$ & 94.0 & 92.6 & 90.1 \\
Upper lip to E-line $(\mathrm{mm})$ & -1.0 & 0.5 & 0.5 \\
Lower lip to E-line $(\mathrm{mm})$ & 1.0 & 1.0 & 1.4 \\
\hline
\end{tabular}

SNA, sella-nasion-A point; SNB, sella-nasion-B point; ANB, A point-nasion-B point; SN, sella-nasion; Go, gonion; Me, menton; U1, upper incisor; L1, lower incisor; Gn, gnathion. 
was discarded.

Molar distalization is an effective treatment approach to relieve crowding, especially to correct Class 11 or 111 molar relationships without affecting the position of the incisors. ${ }^{15-17}$ However, the present patient had a Class 1 molar relationship, indicating the possibility of slight transverse maxillary arch constriction, shown as the crossbite of the left second premolars. Hence, molar distalization was not considered as the primary treatment option.

Extraction was not regarded as a reasonable treatment approach, because the patient showed only mild to moderate crowding. For extraction, closure of excessive space was expected to be challenging with prolonged treatment time.

Correction of the maxillary transverse deficiency by arbitrary arch expansion was seen as risky due to the potential periodontal adverse effects such as alveolar bone dehiscence and gingival recession, especially in the upper left central incisor. ${ }^{18}$ Therefore, expansion by RPE was suggested. Ideal alignment without periodontal compromise can be achieved through orthopedic expansion of the maxilla and relocation of the teeth within the expanded basal bone. Furthermore, RPE allows correction of crossbite and crowding without any considerable effect on the anteroposterior relationship, as desired in this patient.

Although SARPE provides higher predictability, it involves surgical procedures with additional expenses and general risks related to surgery. The patient wished to avoid an invasive treatment. An approach with nonsurgical MARPE was chosen to minimize the undesirable effects of conventional tooth-borne RPE. ${ }^{19}$

The patient was informed of the uncertainty of suture opening and the eventual need for an alternative treatment method and agreed to attempt nonsurgical MARPE.

\section{TREATMENT PROGRESS}

After evaluation of the periodontal status at the peri- odontal department, a customized MARPE appliance using an expander screw with extension plates (KBE; BioMaterials Korea Inc., Seoul, Korea) was fabricated. The MARPE consisted of four bands, connecting arms, an expander body with an expansion screw and four bendable extension plates with insertion holes for the miniscrews. The preliminary assessment of the insertion sites was conducted in the initial cone-beam computed tomography (CBCT) images to determine the anteroposterior position of the expander body to ensure that the palatal bone accommodated the bone screws. The positions of the expander body and the screw holes were marked on the pickup model with four bands. Bands were placed on the second premolars and second molars to avoid injury to the first molars, which had hypersensitivity. The appliance was fabricated by soldering the connecting arms to the bands and contouring the extension plates according to the depth of the palatal vault. The posterior plates were converged toward the midpalatal suture to allow the use of the thickness of the nasal crest. ${ }^{20}$ The MARPE was then cemented on the second premolars and the second molars, and four miniscrews (length, $9.0 \mathrm{~mm}$; diameter, $2.0 \mathrm{~mm}$ for the anterior holes, and length, $7.0 \mathrm{~mm}$ and diameter, $2.0 \mathrm{~mm}$ for the posterior holes; BioMaterials Korea Inc.) were inserted (Figure 3). The selection of the insertion sites and miniscrew dimensions were also based on the norm values of the palatal bone and soft tissue thickness reported in the literature. ${ }^{20,21}$ The anterior miniscrews were inserted in the rugae region, where the palatal bone thickness is known to be sufficient (more than $6 \mathrm{~mm}$ on average). The posterior miniscrews were inserted within $3 \mathrm{~mm}$ from the midpalatal suture, because the bone thickness is known to decrease laterally. ${ }^{21}$

The activation was initiated at a rate of one turn a day (semi-rapid expansion protocol, $0.2 \mathrm{~mm} /$ day). After four weeks of activation, a median diastema was noted clinically (Figure 3). The separation of the midpalatal suture could be confirmed radiographically on a series of periapical views (Figure 4). The activation was completed after a total of 38 turns and $7.6 \mathrm{~mm}$ of MARPE. After
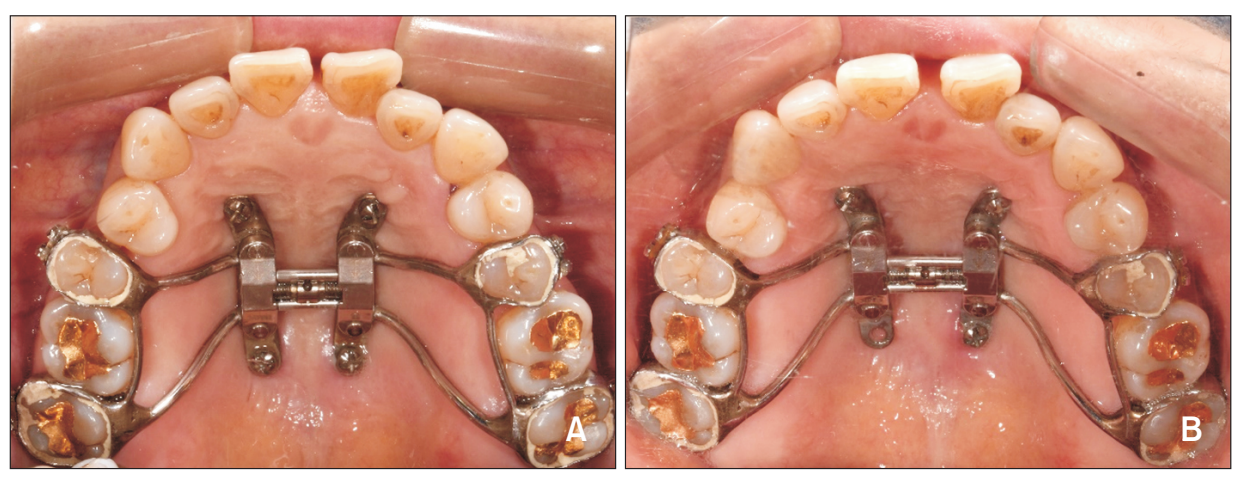

Figure 3. Treatment progress using the miniscrew-assisted rapid palatal expansion appliance. A, Opening of diastema after 4 weeks of activation (0.2 mm/day). B, Post-expansion. 

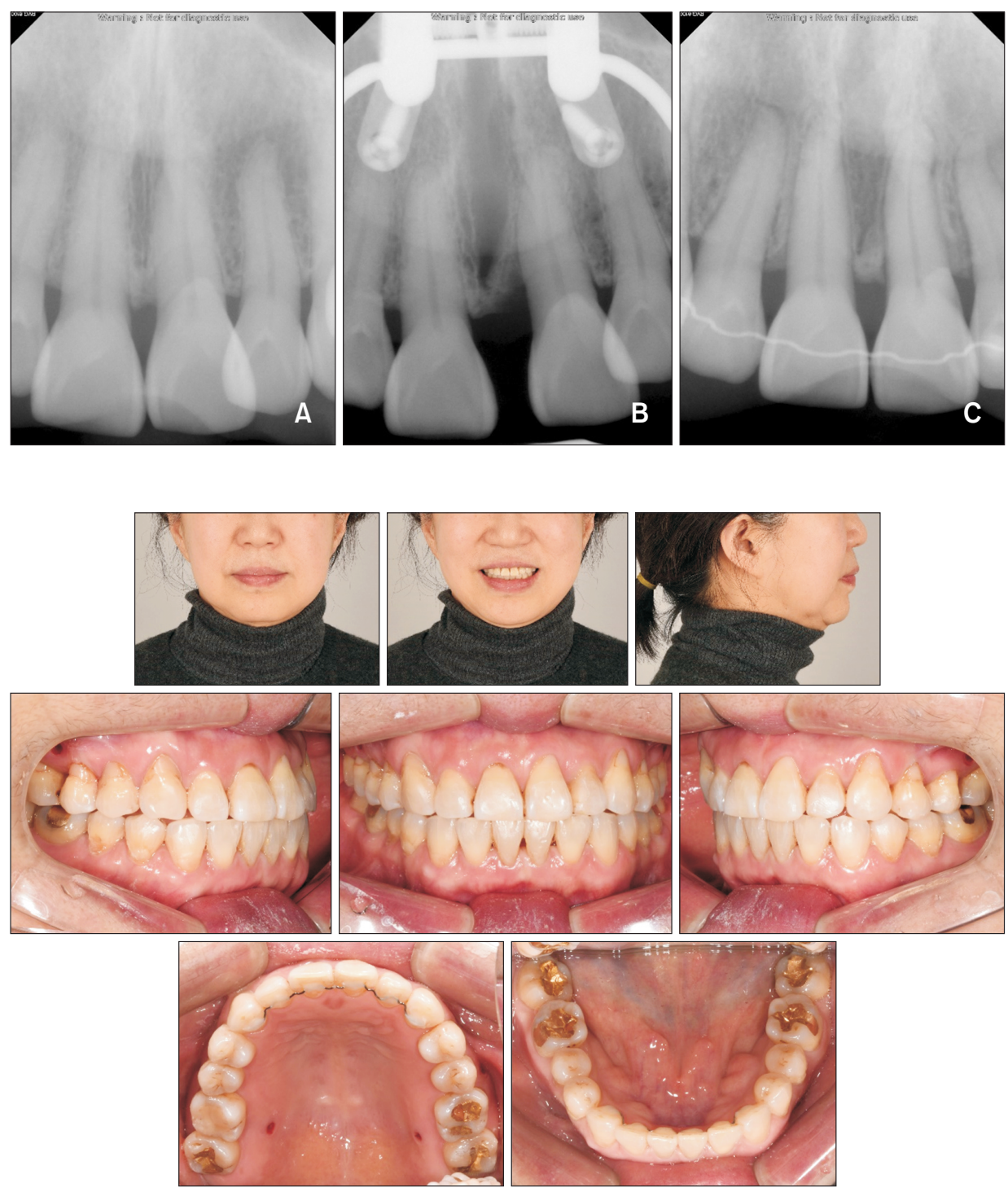

Figure 4. Periapical radiographs. A, Pre-expansion. B, Post-expansion. C, At the end of active treatment.
Figure 5. Final facial and intraoral photographs. completion of expansion, a CBCT scan was obtained.

On the periapical radiographs and CBCT images, a structure resembling a bone-bridge could be observed at the crestal level in the suture gap, indicating a somewhat uneven orthopedic expansion pattern. This structure, however, did not seem to have hindered the suture separation. The post-expansion periapical radiograph showed some vertical alveolar bone loss on the mesial side of the left central incisor. This defect had fully recovered by the end of the active treatment (Figure 4).

After the expansion, 0.018-inch slot self-ligation brackets (Clippy-C; Tomy, Tokyo, Japan) were bonded. The alignment in the mandible was performed directly, while the maxillary alignment was first initiated after two months of consolidation. For the patient's comfort and to secure the transverse dimension of the basal bone during alignment, the MARPE appliance was trans- formed into a passive bone-borne retainer by cutting the arms and removing the bands. In order to further upright the second molars to the lingual side, additional palatal miniscrews were used. All fixed appliances were removed after 12 months of active treatment.

\section{TREATMENT RESULTS}

At the completion of treatment, crossbites were corrected, and acceptable overbite and overjet were established (Figure 5). A Class 1 molar relationship was maintained on both sides, and the maxillary and mandibular crowding were relieved without any considerable change in the profile (Figure 6). The transverse width, measured on CBCT images at the palatal root apex of the maxillary first molars, increased from $31.23 \mathrm{~mm}$ to $34.59 \mathrm{~mm}$ after MARPE (Figure 7). Additionally, the Yonsei Trans- 


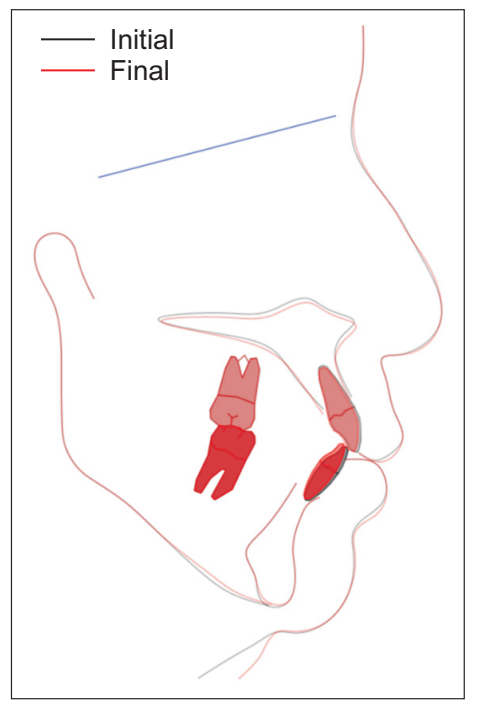

Figure 6. Cephalometric superimposition.

verse Index was measured in order to demonstrate the skeletal change achieved by the expansion. It increased by $2.3 \mathrm{~mm}$ in the maxilla and remained unchanged in the mandible (Figure 7).

Moreover, the opening of the midpalatal suture could be confirmed on the axial palatal section of post-expansion CBCT (Figure 8). A comparison of the pre- and posttreatment dental casts indicated that the maxillary intermolar width increased by $3.0 \mathrm{~mm}$, and the interpremolar width by $4.8 \mathrm{~mm}$ (Figure 9). Despite the adequate amount of expansion, there was no significant change in the clinical crown height of the anchor teeth and the teeth with pre-existing gingival recession (Table 2). Furthermore, comparison of the buccal bone plate at the level of the maxillary first molar on CBCT images before and after expansion did not show any considerable reduction in thickness (Figure 10). The smile esthetics improved, and the patient was satisfied with the outcome.

\section{DISCUSSION}

Adult patients with transverse discrepancies often present conditions unfavorable to periodontal health. Maxillary transverse deficiency is associated with anterior and posterior crossbites and crowding. ${ }^{22}$ Nonaxial loading of the teeth, as a consequence of dental compensation, may result in cervical abfraction. ${ }^{23}$ Such cervical defects can cause apical migration of the gingival margins. Considering these factors, the multiple cervical restorations and gingival recessions found in the present patient may be related to the underlying transverse problems.

The initial alveolar support and clinical gingival margins were not significantly detrimental. However, consid- ering the age of the patient, a safe treatment modality was needed. In order to ensure periodontal health, transverse correction by orthopedic expansion was suggested, despite the uncertainty of successful suture opening.

The present case describes, to the best of our knowledge, nonsurgical palatal expansion in the oldest patient reported in the literature. ${ }^{24}$ The general consensus has been that orthopedic palatal expansion by nonsurgical means is feasible only until adolescence. ${ }^{10,25}$ The mechanical resistance resulting from increasing interdigitation of the facial sutures is commonly believed to limit orthopedic expansion in mature patients..$^{8-11}$ On the other hand, the regulatory mechanism behind the fusion of facial sutures differs from that of calvarial sutures. ${ }^{12,26}$ According to a previous study, cranial sutures are obliterated in the absence of dura mater. ${ }^{27}$ The lack of such biochemical modulation by dura mater in the facial sutures may enable nonsurgical expansion even in elderly patients with interdigitated sutures, as long as they are not fused at the histologic level.

Unfortunately, no reliable guidelines have been proposed to predicting the success of skeletal expansion in nongrowing patients. According to Wehrbein and Yildizhan, ${ }^{28}$ besides the factors related to the midpalatal suture itself, the complexity and patency of pterygomaxillary junction and other circumaxillary sutures may affect the outcome. Because it is technically not possible to examine all affecting sutures, the only reliable method to confirm suture patency is an actual clinical trial of orthopedic expansion.

For these reasons, nonsurgical orthopedic expansion was attempted in the present patient. In order to reduce the adverse effects such as buccal tipping of the anchor teeth and thinning of the buccal bone plate, a MARPE appliance was used. Previous studies have shown that the incorporation of miniscrews in palatal expanders reduces dental side effects, but does not completely eliminate them. ${ }^{19}$ Moreover, in the present case, the second molars showed some dental tipping, which caused an intermediate occlusal interference and overall open bite. However, the open bite spontaneously subsided following the uprighting movement of the molars, and desirable transverse occlusion was obtained after treatment (Figure 5). Eventually there was no significant change in the overall facial vertical dimension (Figure 6).

Regarding the frequency of activation, a semi-rapid expansion protocol $(0.2 \mathrm{~mm} /$ day $)$ was used to allow enough time for remodeling of the interdigitated suture structure and to monitor the periodontal changes in the buccal plates of the anchor teeth. Considering the inherent complexity of the interdigitation along the suture and the uncertainty in orthopedic expansion due to the possible synostosis in adults, ${ }^{26}$ careful monitoring is crucial. Abrupt expansion may lead to unfavorable fracture 

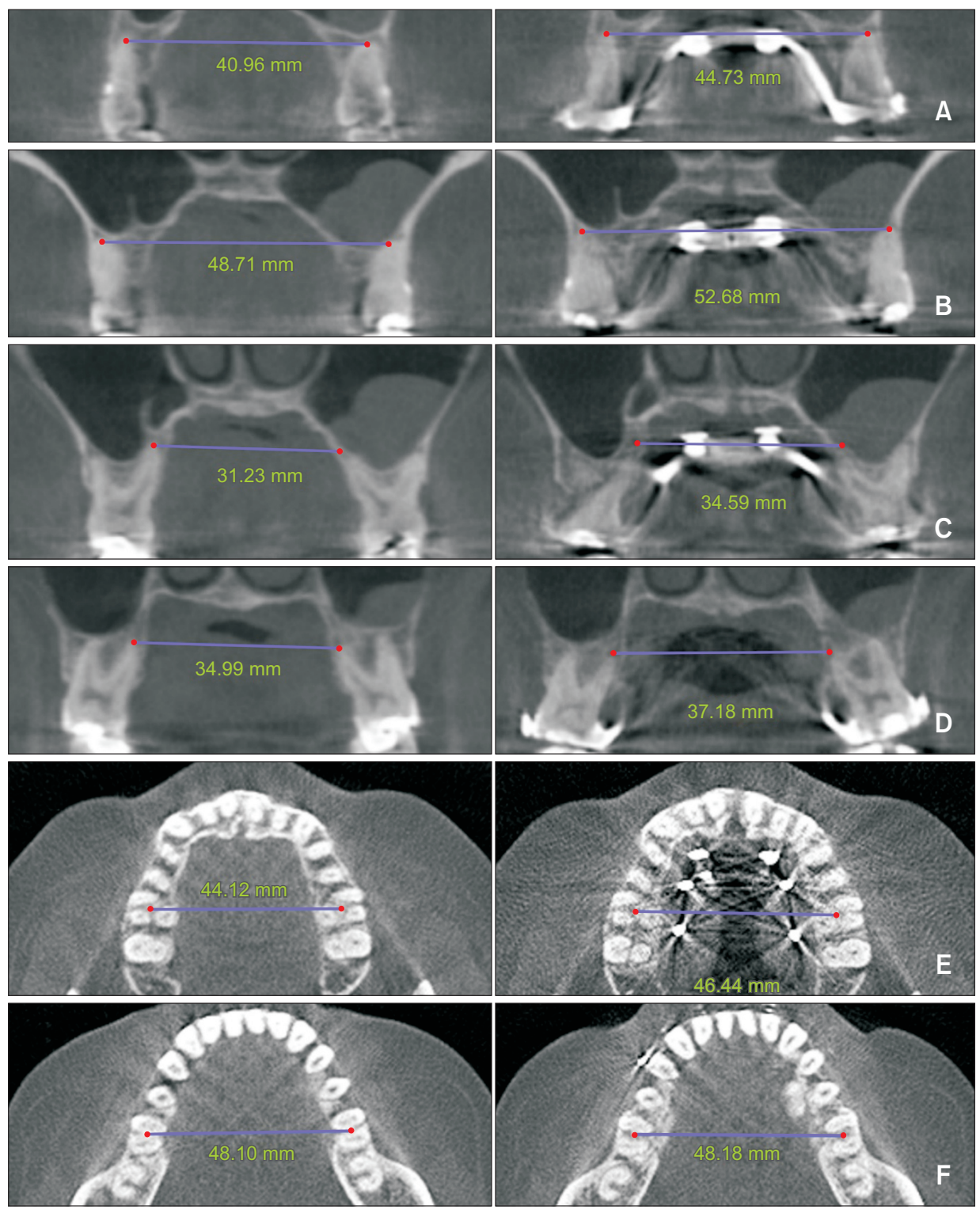

Figure 7. Interapex width before and after palatal expansion measured between the maxillary first premolars (A); second premolars (B); first molars (C); second molars (D); and Yonsei Transverse Index measured before and after palatal expansion in the maxilla (E) and mandible (F).
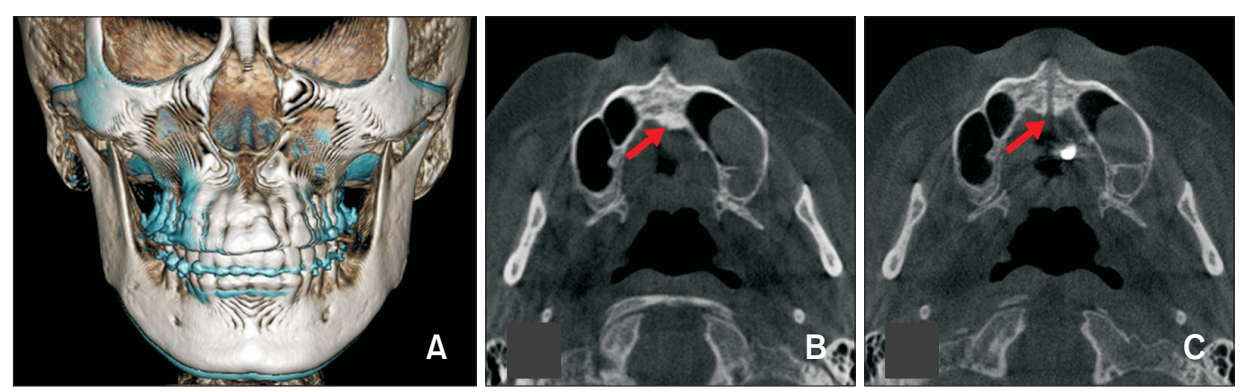

Figure 8. Comparison of cone-beam computed tomography (CBCT) images before and after palatal expansion. A, Superimposition of pre- and post-expansion CBCT scans. B, Axial palatal section before expansion. C, Axial view showing suture opening after expansion. 

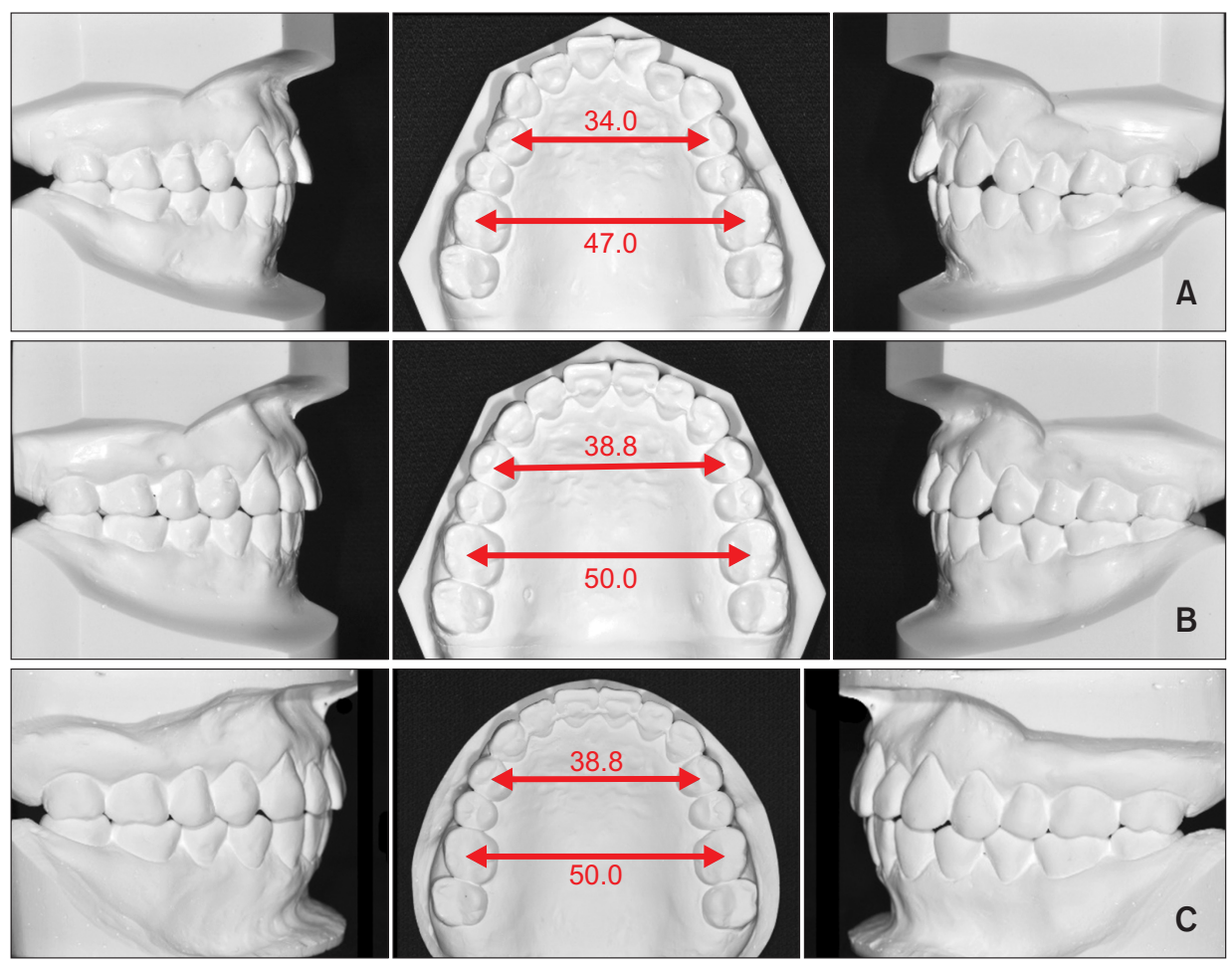

Figure 9. Comparison of pretreatment $(A)$, posttreatment (B), and follow-up (C) dental casts.

Table 2. Clinical crown heights of the maxillary teeth before and after treatment

\begin{tabular}{lccc}
\hline \multicolumn{1}{c}{ Tooth } & Pretreatment $(\mathbf{m m})$ & Posttreatment $\mathbf{( m m})$ & 6-month follow-up $(\mathbf{m m})$ \\
\hline Right canine & 10.7 & 10.6 & 10.5 \\
Right first premolar & 9.3 & 9.2 & 9.3 \\
Right second premolar & 8.9 & 8.7 & 8.9 \\
Right first molar & 7.5 & 7.6 & 7.6 \\
Right second molar & 7.1 & 7.0 & 7.0 \\
Left canine & 11.2 & 11.2 & 11.3 \\
Left first premolar & 9.8 & 9.8 & 9.9 \\
Left second premolar & 8.0 & 8.0 & 8.1 \\
Left first molar & 7.9 & 7.5 & 7.5 \\
Left second molar & 6.1 & 6.2 & 6.1 \\
\hline
\end{tabular}

or bony dehiscence, so a modest expansion rate was supposed to be reliable. Moreover, studies with experimental animal models have demonstrated that constant pressure to the suture may lead to compression osteogenesis, which causes changes in the suture structure. ${ }^{29}$ To allow this process to occur, it is presumably important to provide enough time for bone remodeling, rather than applying greater force by activating the screw with shorter intervals. Overall, orthopedic palatal expansion in aged individuals requires careful observation of the changes in the buccal plates, midpalatal suture, dental tipping, and general occlusion as well as the patient response. Considering these aspects, a semi-rapid protocol may be relatively safer in older patients with highly interdigitated facial sutures.

The use of MARPE in the present case yielded satisfactory outcomes with good maintenance of gingival health. The bone-bridge observed after the expansion is possibly due to the tight interdigitation in the incisal interproximal crestal area, which seemed to have caused some bone bending on the mesial side of the left central incisor. Nevertheless, the opening of the suture was relatively symmetric and constant along the midpalatal suture, indicating an overall orthopedic expansion of the maxilla. In relation to this orthopedic expansion, the intermolar width increased by $3.0 \mathrm{~mm}$ and the interpre- 


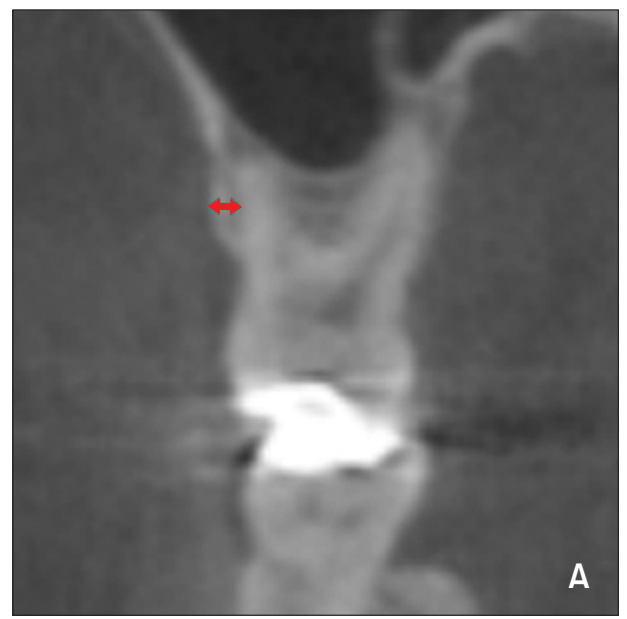

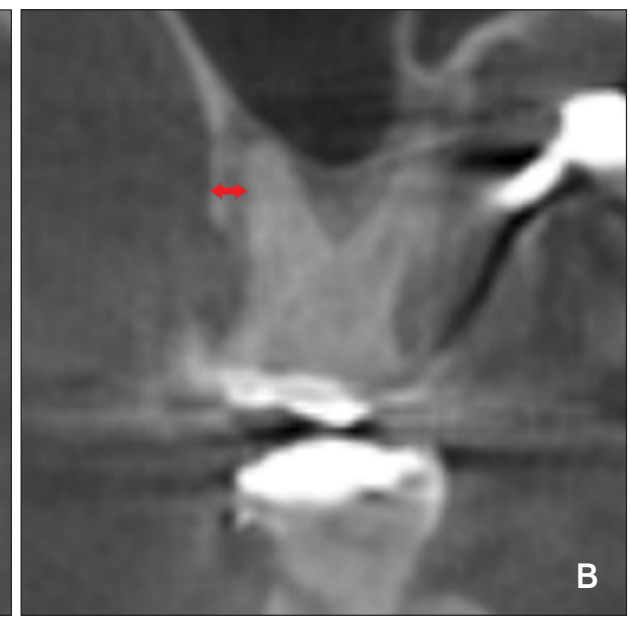

B
Figure 10. Buccal bone plate before (A) and after (B) palatal expansion.

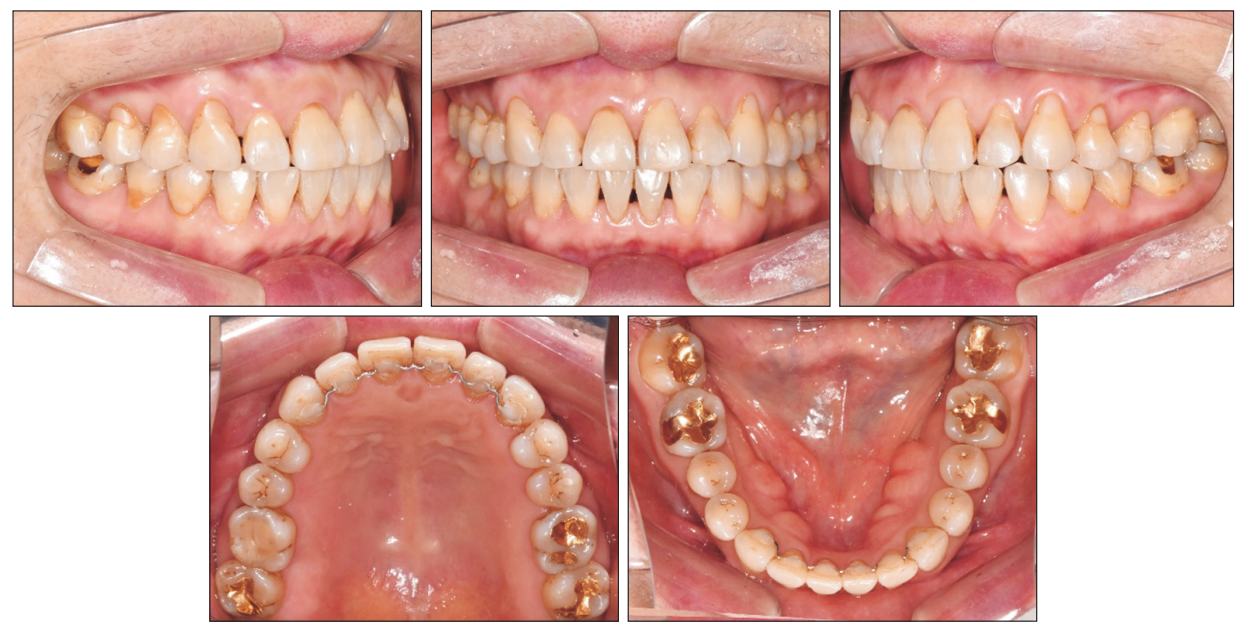

Figure 11. Intraoral photographs taken at the 6-month follow-up. molar width increased by $4.8 \mathrm{~mm}$ without any significant buccal tipping. The treatment change, although relatively small, approximated the average amount of expansion reported by Choi et al. ${ }^{6}$ and is considered meaningful since the movement pattern can be defined as bodily displacement of the posterior segment against the thin buccal plate. Since an increase of more than $4 \mathrm{~mm}$ in the interpremolar width is possibly associated with gingival recession, ${ }^{30}$ the use of MARPE in the present case can be justified.

The occlusion and transverse width remained stable, and gingival health was maintained during the followup period of six months (Figure 11). According to Reitan $^{31}$ the periodontal fibers under orthodontic force would remain stretched after treatment, which may lead to clinical changes in the periodontal tissue. Therefore, we presumed that observation of the clinical changes in the gingival tissue within a relatively short term would be crucial to detect some adverse effects of the expansion in the present case. A longer follow-up period is necessary to evaluate the long-term stability of the oc- clusion. However, long-term gingival changes may also reflect individual lifestyle factors such as brushing and mastication habits. ${ }^{32}$ Thus, proper orthopedic expansion in aged individuals may be an option for preservation of the periodontal structures on the buccal side.

\section{CONCLUSION}

Nonsurgical orthopedic expansion of the maxilla was achieved in a 60-year-old patient with the help of a MARPE appliance. This approach allowed the correction of crossbites and crowding without negatively affecting the pre-existing gingival recession. This case does not represent the general clinical observations. However, it is a good example demonstrating that advanced age is not an absolute contraindication for nonsurgical RPE. Even in elderly patients, orthopedic expansion can be a reasonable option and may eventually deliver satisfactory results, as shown in the present case. 


\section{CONFLICTS OF INTEREST}

No potential conflict of interest relevant to this article was reported.

\section{REFERENCES}

1. Atik E, Taner T. Stability comparison of two different dentoalveolar expansion treatment protocols. Dental Press J Orthod 2017;22:75-82.

2. Papapanou PN, Wennström JL, Gröndahl K. Periodontal status in relation to age and tooth type. A cross-sectional radiographic study. J Clin Periodontol 1988;15:469-78.

3. Van der Velden U. Effect of age on the periodontium. J Clin Periodontol 1984;11:281-94.

4. Angell EH. Treatment of irregularities of the permanent or adult teeth. In: White JD, McQuillen JH, Ziegler GJ, White JW, Kirk EC, Anthony LP, eds. The dental cosmos. Philadelphia: S.S. White Dental Manufacturing Company; 1860. p. 540-4, 99-600.

5. Haas AJ. The treatment of maxillary deficiency by opening the midpalatal suture. Angle Orthod 1965; 35:200-17.

6. Choi SH, Shi KK, Cha JY, Park YC, Lee KJ. Nonsurgical miniscrew-assisted rapid maxillary expansion results in acceptable stability in young adults. Angle Orthod 2016;86:713-20.

7. Lee KJ, Park YC, Park JY, Hwang WS. Miniscrew-assisted nonsurgical palatal expansion before orthognathic surgery for a patient with severe mandibular prognathism. Am J Orthod Dentofacial Orthop 2010; 137:830-9.

8. Persson M, Thilander B. Palatal suture closure in man from 15 to 35 years of age. Am J Orthod 1977; 72:42-52.

9. Melsen B. Palatal growth studied on human autopsy material. A histologic microradiographic study. Am J Orthod 1975;68:42-54.

10. Shetty V, Caridad JM, Caputo AA, Chaconas SJ. Biomechanical rationale for surgical-orthodontic expansion of the adult maxilla. J Oral Maxillofac Surg 1994;52:742-9; discussion 750-1.

11. Asscherickx K, Govaerts E, Aerts J, Vande Vannet B. Maxillary changes with bone-borne surgically assisted rapid palatal expansion: a prospective study. Am J Orthod Dentofacial Orthop 2016;149:374-83.

12. Kokich VG. Age changes in the human frontozygomatic suture from 20 to 95 years. Am J Orthod 1976;69:411-30.

13. Uysal T, Memili B, Usumez S, Sari Z. Dental and alveolar arch widths in normal occlusion, class $11 \mathrm{di}-$ vision 1 and class 11 division 2. Angle Orthod 2005; 75:941-7.
14. Koo YJ, Choi SH, Keum BT, Yu HS, Hwang CJ, Melsen B, et al. Maxillomandibular arch width differences at estimated centers of resistance: comparison between normal occlusion and skeletal Class 111 malocclusion. Korean J Orthod 2017;47:167-75.

15. Jang W, Shin C, Hwang S, Kim KH, Jackson T, Nguyen $\mathrm{T}$, et al. Nonsurgical treatment of an adult with a skeletal Class 111 malocclusion combined with a functional anterior shift, severely overclosed vertical dimension, and a reverse smile. Am J Orthod Dentofacial Orthop 2020;157:561-70.

16. Choi NC, Park YC, Lee HA, Lee KJ. Treatment of Class 11 protrusion with severe crowding using indirect miniscrew anchorage. Angle Orthod 2007; 77:1109-18.

17. Bechtold TE, Kim JW, Choi TH, Park YC, Lee KJ. Distalization pattern of the maxillary arch depending on the number of orthodontic miniscrews. Angle Orthod 2013;83:266-73.

18. Kraus CD, Campbell PM, Spears R, Taylor RW, Buschang $\mathrm{PH}$. Bony adaptation after expansion with light-to-moderate continuous forces. Am J Orthod Dentofacial Orthop 2014;145:655-66.

19. Seong EH, Choi SH, Kim HJ, Yu HS, Park YC, Lee KJ. Evaluation of the effects of miniscrew incorporation in palatal expanders for young adults using finite element analysis. Korean J Orthod 2018;48:81-9.

20. Wehrbein H, Merz BR, Diedrich P. Palatal bone support for orthodontic implant anchorage--a clinical and radiological study. Eur J Orthod 1999;21:65-70.

21. Kang S, Lee SJ, Ahn SJ, Heo MS, Kim TW. Bone thickness of the palate for orthodontic mini-implant anchorage in adults. Am J Orthod Dentofacial Orthop 2007;131(4 Suppl):S74-81.

22. McNamara JA. Maxillary transverse deficiency. Am J Orthod Dentofacial Orthop 2000;117:567-70.

23. Nascimento MM, Dilbone DA, Pereira PN, Duarte WR, Geraldeli S, Delgado AJ. Abfraction lesions: etiology, diagnosis, and treatment options. Clin Cosmet Investig Dent 2016;8:79-87.

24. Moon W. Maxillary expansion in skeletally mature patients with TADs. In: Park JH, ed. Temporary anchorage devices in clinical orthodontics. Hoboken: John Wiley \&t Sons, Inc.; 2020.

25. Proffit WR, Fields HW, Sarver DM. Contemporary orthodontics. St. Louis: Elsevier Health Sciences; 2014.

26. Cohen MM Jr. Sutural biology and the correlates of craniosynostosis. Am J Med Genet 1993;47:581616.

27. Opperman LA, Sweeney TM, Redmon J, Persing JA, Ogle RC. Tissue interactions with underlying dura mater inhibit osseous obliteration of developing cranial sutures. Dev Dyn 1993;198:312-22.

28. Wehrbein H, Yildizhan F. The mid-palatal suture in 
young adults. A radiological-histological investigation. Eur J Orthod 2001;23:105-14.

29. Castello JR, Olaso AS, Chao JJ, McCarthy JG, Molina F. Craniofacial shortening by contraction osteogenesis: an experimental model. Plast Reconstr Surg 2000;105:617-25; discussion 626-7.

30. Garib DG, Henriques JF, Janson G, de Freitas MR, Fernandes AY. Periodontal effects of rapid maxillary expansion with tooth-tissue-borne and tooth-borne expanders: a computed tomography evaluation. Am J Orthod Dentofacial Orthop 2006;129:749-58.

31. Reitan K. Tissue rearrangement during retention of orthodontically rotated teeth. Angle Orthod 1959; 29:105-13.

32. Kassab MM, Cohen RE. The etiology and prevalence of gingival recession. J Am Dent Assoc 2003; 134:220-5. 